\title{
Strategie discorsive in spagnolo L1 ed L2 a confronto: un'indagine su corpora dialogici ${ }^{1}$
}

\author{
Iolanda Alfano, Renata Savy, Simona Sbranna, Loredana \\ Schettino \\ Università degli Studi di Salerno
}

According to previous studies on L1 Italian and Spanish, speakers prefer different pragmatic strategies and adopt specific pragmatic patterns to express their attention to the interlocutor. This study deals with communicative strategies used in dialogic speech in L1 and L2 Spanish considering both textual structure and interaction between the two interlocutors. More in detail, the aim of this research is to analyse how speakers introduce and manage Discourse Topics in order to compare the behaviour of native and non-native Spanish speakers. The study examines two corpora of task-oriented dialogues, the corpus DiESPA, of Peninsular Spanish L1 -collected in different geographic areas- and the corpus DiELE-(I), of Spanish L2 -collected in the University of Salerno with Italian learners of a CEFR level B2-C1. The analysis of Discourse Topics introduction and management allows defining crucial textual characteristics and defining speakers' attitudes towards interlocutors. From a qualitative and a quantitative analysis, which examines the percentage of occurrences of pragmatic moves used by native and non-native speakers, it emerges that differences in native speakers and L2 learners' strategic choices used to complete the task are due to their limited linguistic competence, especially grammatical and lexical, rather than to pragmatic and cultural factors.

Keywords: L1/L2 Spanish, dialogic speech, discourse strategies, textual structure

\footnotetext{
${ }^{1}$ L'intero lavoro è frutto della collaborazione tra le autrici, che compaiono in puro ordine alfabetico. Tuttavia, esclusivamente ai fini delle procedure di valutazione scientifica, a Iolanda Alfano si deve la stesura dei $\S 1$ e 2; a Renata Savy quella dei $\S 5$ e 6; a Simona Sbranna quella del $\$ 4$ e a Loredana Schettino quella del § 3.
} 


\section{Introduzione e obiettivi}

Studi precedenti sulle strategie discorsive condotti in chiave contrastiva mettono in luce il peso del contesto socio-culturale sulle dinamiche di interazione: lingue diverse mostrano tratti variabili nell'accordare preferenze e "dispreferenze" alle diverse scelte pragmatiche e nell'assumere atteggiamenti e modalità di conduzione dello scambio dialogico (House 2006; Bazzanella 2015).

In particolare, il confronto tra lo spagnolo e l'italiano mostra una diversa attitudine sia nella conduzione del dialogo che nell'interazione con l'interlocutore, nonché preferenze diverse nella gamma delle strategie discorsive impiegate: in spagnolo si riscontra una predilezione per strategie che coinvolgono l'interlocutore e sollecitano la sua collaborazione più di quanto accada in italiano. Le diverse strategie comunicative impiegate in italiano, invece, hanno in comune la caratteristica di mettere al centro il punto di vista del parlante che tiene il turno (Savy \& Solís 2008; Solís \& Savy 2012; Savy \& Alfano in press).

Tanto premesso, lo scopo principale di questa ricerca consiste nell'indagare se e in che misura apprendenti italofoni di spagnolo L2 tendano a ripetere gli schemi adottati nella loro lingua materna (l'italiano) o, invece, mostrino gradi di avvicinamento alle strutture preferite nella lingua target (lo spagnolo).

Impiegando tecniche e metodologie della Corpus Linguistics, ci proponiamo dunque di verificare quanto profondamente la competenza pragmatica acquisita come parte della competenza grammaticale in una lingua straniera plasmi i comportamenti verbali e su quali piani.

Tale contributo costituisce uno studio pilota nel quale esaminiamo un piccolo corpus di dialoghi, con lo scopo di sperimentare una metodologia di analisi e suggerire strumenti interpretativi per analisi successive. Le dimensioni ridotte del corpus, così come l'assenza di analisi statistiche ad esse legata, fanno sì che questo lavoro costituisca un'indagine preliminare, che non consente certamente di giungere a generalizzazioni, ma che ha, piuttosto, un valore esplorativo. Le considerazioni cui giungiamo, pertanto, dovranno essere verificate su un corpus di maggiore ampiezza, considerando in maniera sistematica le variabili in gioco e la loro influenza sulle scelte strategiche dei parlanti.

Come vedremo, l'analisi pragmatica si incentra su un particolare dominio della struttura discorsiva, l'introduzione e la gestione di un argomento di discorso (o Discourse Topic, DT, § 2), che dà luogo ad una transaction (Sinclair \& Coulthard 1975; Anderson et al. 1991; Isard \& Carletta 1995; Malouf 1995; Carletta et al. 1996). Sul piano testuale, la presenza di un DT origina una macrostruttura che si snoda in vari sub-topic, strettamente legata a fattori di cotesto e contesto; 
sul piano interazionale, le modalità con le quali il parlante introduce, negozia e maneggia un DT riflettono scelte di maggiore o minore disponibilità allo scambio.

\section{Dominio d'analisi: il DT e la struttura discorsiva}

\subsection{Il DT in letteratura}

La nozione di DT è stata elaborata in ambiti diversi e impiegata in vari modi. Tutti coloro che si sono occupati del tema hanno, in primo luogo, sottolineato la sua vaghezza e difficoltà di identificazione. Si tratta, infatti, di una nozione complessa e legata a concetti altrettanto articolati, quali la datità, la recuperabilità, l'accessibilità e lo stato di attivazione di un referente (Gundel 1988, 2003; Gundel et al. 1993; Lambrecht 1994; Lambrecht \& Michaelis 1998).

Tra i principali punti di vista, possiamo riconoscere i contributi provenienti dall'ambito dell'Analisi della Conversazione e del Discorso (Brown \& Yule 1983), della Pragmatica Formale (Kuppevelt 1995; Roberts 1996; Büring 1997; Beyssade \& Marandin 2002), della Pragmatica Cognitiva (Chang 2007) e infine della Semantica Formale (Asher 2004).

Nell'ambito dell' Analisi della Conversazione e del Discorso, il DT è definito come l'argomento del discorso nel suo complesso (senza specificare, però, se si tratti di un intero discorso o solo una parte di esso) ed è, quindi, legato al concetto di aboutness (Brown \& Yule 1983). Sebbene sia difficile individuare una definizione univoca, il DT si configura come un'entità globale, definibile solo in relazione all'intero discorso ${ }^{2}$. I vari DT sono negoziati all'interno della conversazione ed è possibile riconoscere tra loro dei rapporti gerarchici.

Nella prospettiva della Pragmatica Formale, van Kuppevelt (1995) considera la topicalità come il principale principio organizzativo della struttura del discorso, facendone il centro di un procedimento induttivo di domande definito questioning. In tale proposta si considera, infatti, che dietro ciascun topic vi sia una domanda (implicita o esplicita) e che esista una gerarchia tra domande autonome su topic di ordine superiore (main constituting questions) e domande, non autonome, che si generano da una risposta insoddisfacente ad una precedente domanda (sub-topic

\footnotetext{
${ }^{2}$ In tal senso, la nozione di DT si configura in opposizione a quella di Sentence Topic (ST), concetto elaborato non nel dominio del discorso, bensì a livello di frase. Tra le accezioni più largamente condivise di ST ricordiamo quelle di Gundel (1988) e Lambrecht (1994), il quale distingue la nozione pragmatica di referente dall'espressione linguistica del ST quale costituente (che può poi esprimere il referente). Non ci soffermiamo sulla nozione di ST e sulle differenze con altre unità informative, per le quali rimandiamo alle rassegne di De Leo (2008) e Chini (2010).
} 
constituting sub-questions). Il DT è poi concepito come un topic di ordine ancora superiore, inteso come l'insieme dei topic di una domanda autonoma. La relazione question/answer è alla base anche di altre proposte dello stesso ambito (Roberts 1996; Büring 1997; Beyssade \& Marandin 2002).

Dal punto di vista della Pragmatica cognitiva, l'identificazione del DT avviene mediante processi inferenziali: il DT va ben al di là dei significati letterali, prescinde dalle caratteristiche grammaticali e morfosintattiche e può comporsi di materiale non enunciato esplicitamente (Chang 2007).

Nella visione della Semantica Formale, invece, la funzione del DT maggiormente messa in luce è quella di connessione tra le varie parti del discorso. Infine, è altresì sottolineata l'importanza dei diversi casi e contesti conversazionali: "Discourse topics have different roles to play in different constructions. [...] It seems not wise to search for a completely general notion, as different discourse relations make different demands on what topics should do" (Asher 2004: 200).

I vari approcci, dunque, evidenziano proprietà diverse di ciò che un DT designa. Per alcuni (analisti del discorso e della conversazione), il tratto pertinente per la sua definizione è direttamente connesso alla proprietà dell' aboutness. Per altri (pragmatisti formali), la nozione di DT, e di conseguenza la strutturazione del discorso, è fortemente legata alla modulazione question/answer, come frutto di domande. In un certo senso, tali prospettive tengono in gran conto gli aspetti contestuali e cotestuali e considerano meno quelli cognitivi e inferenziali. Gli approcci pragmatico-cognitivi e semantici, invece, danno meno rilievo al fattore contesto e fondano le loro proposte sulla relazione con i processi inferenziali, sottolineando una certa indipendenza dagli aspetti più propriamente grammaticali.

Negli ambiti che abbiamo menzionato, quindi, un DT è certamente concepito in maniera molto diversa e spesso la sua definizione risulta piuttosto generica e vaga. Tuttavia, è possibile riconoscere alcune fondamentali caratteristiche testuali del DT comuni ai diversi punti di vista.

\subsubsection{Caratteristiche del DT}

La struttura testuale è, in qualche modo, riconosciuta unanimemente come una struttura di tipo gerarchico, nella quale esiste un argomento principale da cui si generano e dipendono nel discorso vari sub-topic (come vedremo, anch'essi snodabili, a loro volta, a diversi livelli gerarchici).

In primo luogo, quindi, possiamo affermare che il DT:

- è sovraordinato ad altre entità e

- si realizza nel dominio del discorso (diversamente dal Sentence Topic). 
Il diverso dominio non costituisce però certamente l'unica differenza con il Sentence Topic. Quest'ultimo, infatti, è sempre esplicitamente espresso, mentre il DT:

- può comporsi di materiale non esplicito e non ha alcuna corrispondenza prestabilita con materiale linguistico.

Indipendentemente poi dalle diverse concezioni della nozione, l'identificazione del DT:

- è indissolubilmente legata a contesto e cotesto e

- assicura coerenza e coesione al discorso.

Tutte le proposte, infine, sottolineano l'indipendenza della nozione da aspetti puramente grammaticali e il suo legame con i processi cognitivi e inferenziali. Quindi, concludendo, un DT:

- prescinde dagli aspetti grammaticali e

- dipende dai processi cognitivi e inferenziali.

Tutte queste proprietà fanno sì che le sue caratteristiche precipue dipendano dalla conversazione in cui si inserisce.

\subsection{Il DT nella nostra analisi}

Nel nostro lavoro, il contesto conversazionale è determinato da una situazione gioco, all'interno di un dialogo task-oriented (\$ 3.1). Ci troviamo, quindi, nel caso di un particolare tipo di interazione elicitata mediante una tecnica di pilotaggio dello scambio, in cui i parlanti condividono uno scopo ben preciso da raggiungere (Sinclair \& Coulthard 1975) ${ }^{3}$. Svolgono un compito che richiede cooperazione e collaborazione, mirata, appunto, alla risoluzione del gioco proposto ${ }^{4}$.

\footnotetext{
${ }^{3}$ Sebbene l'accezione impiegata nell'ambito della Corpus Linguistics faccia riferimento ad una tecnica ben precisa, qualunque conversazione potrebbe essere considerata task-oriented, nel senso che ha un suo task che struttura lo scambio dialogico. Ad esempio, si può considerare il caso di una telefonata per pianificare un'uscita serale: la conversazione è strutturata in funzione del task prendere un appuntamento per uscire e articolata intorno a vari argomenti che, come vedremo più avanti, risultano centrali nella nostra analisi (per esempio, quando incontrarsi, dove e come trascorrere la serata, se e a chi estendere l'invito, ecc.).

${ }^{4}$ Grazie a tali tecniche, è possibile controllare vari tipi di fattori extralinguistici. Innanzitutto, si riduce la variabilità relativa alla situazione comunicativa. In secondo luogo, è possibile selezionare i parlanti in relazione agli obiettivi della ricerca, nonché stabilire e quindi controllare il
} 
La struttura dialogica risultante, pur essendo variabile e complessa, si intesse intorno a vari blocchi tematici, definiti transaction (cfr. Sinclair \& Coulthard 1975). Tale nozione viene ripresa dall'HCRC di Edimburgo nello schema Map-task (Anderson et al. 1991; Isard \& Carletta 1995; Carletta et al. 1996, 1997), mediante il quale, di fatto, all'apertura di una transaction corrisponde l'introduzione di un nuovo topic, inteso come referente di un elemento della mappa. Tuttavia, nella definizione degli autori non è esplicitamente indicato un rapporto tra topic e transaction. Malouf (1995: 4), invece, dichiara in maniera esplicita che "A transaction corresponds more or less to a single topic-unit", legando quindi il concetto di transaction a quello di topic e, di conseguenza, l'apertura di una transaction all'introduzione di un nuovo topic.

Riprendendo tale legame, ci riferiamo ad un DT come ad un argomento con un suo sviluppo nello scambio comunicativo e a una transaction, considerandola come il segmento su cui verte un DT.

I dialoghi task-oriented costituiscono un terreno privilegiato d'analisi della struttura testuale di un DT, in quanto presentano un particolare tipo di struttura conversazionale e consentono di seguire in maniera dettagliata lo snodarsi di un DT. Il gioco proposto, il task, che assume la funzione decisiva di contesto conversazionale, determina gli argomenti di conversazione. La struttura del discorso che ne risulta è plasmata dal compito da svolgere e si sviluppa in maniera peculiare sull'esplorazione di alcuni argomenti, che assumono la forma e la funzione di DT. La nozione di DT è quindi centrale nell'analisi testuale di tali tipi di scambi (De Leo 2008).

\subsubsection{Gerarchie tra le entità topicali}

La struttura topicale è organizzata e concatenata gerarchicamente. Esiste un Topic sovraordinato (overarching topic, Prévot 2001) che incorpora tutti gli altri: ogni dialogue topic può dare origine a uno o più sub-dialogue aventi uno o più sub-

\footnotetext{
tipo di relazione che intercorre tra gli interlocutori, sia in termini di familiarità che di simmetria. Dal punto di vista lessicale e morfosintattico, tali metodi facilitano specifiche produzioni linguistiche mediante un'opportuna selezione di nomi, magari attribuiti ad hoc ad alcuni referenti del gioco. Infine, consentono un'osservazione dei meccanismi conversazionali che intervengono nell'interazione comunicativa a diversi livelli di analisi e in relazione a tutto un insieme di fenomeni comunicativi. Tuttavia, sebbene la situazione gioco risulti utile per distrarre i partecipanti dalla condizione di registrazione, favorendo naturalezza e spontaneità di espressione, è altresì indiscutibile che tali tecniche presentino anche degli svantaggi. Il parlato che si ottiene, definito appunto "semi-spontaneo" è, infatti, chiaramente vincolato alla necessità di portare a termine un compito preciso e risulta, quindi, per certi versi, meno variabile e più prevedibile rispetto ad una conversazione completamente spontanea e libera.
} 
topic. Nella gestione del dialogo non tutti gli argomenti trattati assumono valenza topicale, ma solo quelli accettati da entrambi i partecipanti e sviluppati fino alla risoluzione e che vanno, quindi, a costituire il commonground della conversazione.

Nello sviluppo di ciascun DT è possibile riconoscere varie fasi: uno dei partecipanti propone un argomento. Se non viene accettato, sarà seguito da una nuova proposta. Se invece viene accettato, sarà gestito per un certo lasso di tempo, dando luogo a potenziali gerarchie o catene di sub-topic, anche a diversi livelli gerarchici. Successivamente, il DT sarà poi esaurito, e quindi chiuso, per permettere l'attivazione di un nuovo DT. La conversazione si conclude quando i parlanti interrompono l'interazione; ciascun discorso interno alla conversazione si chiude, invece, con l'esaurirsi dell'argomento.

Dunque, sul piano conversazionale, i dialoghi task-oriented risultano organizzati in transaction intorno a vari DT.

Come vedremo ( $\$ 3.2)$, le modalità di introduzione e di gestione di un DT possono essere molto diverse e rispondono a precise scelte strategiche dei parlanti.

\section{Materiali e metodi}

\subsection{Corpus}

Il corpus analizzato proviene da due raccolte indipendenti di dialoghi task-oriented: il corpus DiESPA comprende 16 dialoghi prodotti da nativi spagnoli, raccolti a Barcellona, Siviglia, Almeria e Malaga; il corpus DiELE-(I), è costituito dallo stesso tipo di dialoghi raccolti all'Università di Salerno impiegando apprendenti di spagnolo di livello B2-C1 del QCER ${ }^{5}$.

Quattro dialoghi sono stati elicitati mediante la tecnica del "Test delle differenze" (Péan et al. 1993; Cerrato 2007): due persone, sedute di spalle e utilizzando, quindi, esclusivamente il canale verbale, hanno il compito di individuare alcune differenze tra due vignette che differiscono per alcuni particolari (Figura $1)^{6}$.

\footnotetext{
${ }^{5}$ Entrambi i corpora sono in costante aggiornamento. Il primo, il corpus DiESPA, è già disponibile all'indirizzo www.parlaritaliano.it nella sezione "Corpora di parlato".

${ }^{6}$ Le vignette impiegate appartengono al Progetto CLIPS (Savy \& Cutugno 2009).
} 


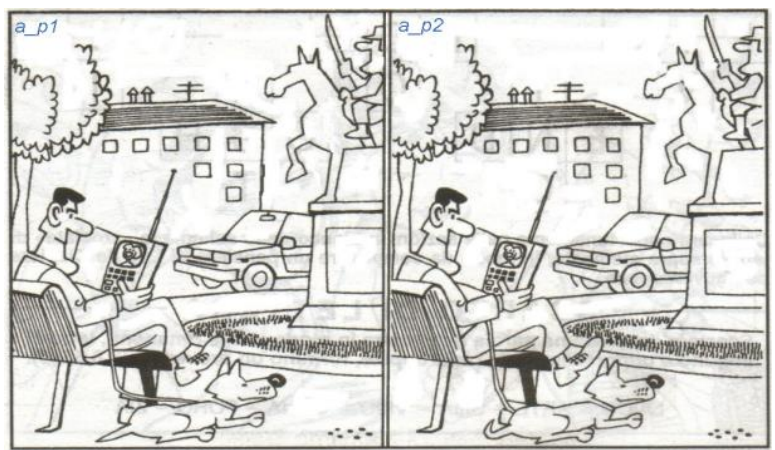

Figura 1. Vignette impiegate per l'elicitazione dei dialoghi (da Cutugno 2007)

Il corpus analizzato contiene un totale di 135 occorrenze di transaction, distribuite in spagnolo L1 e L2 come illustrato in Tabella $1^{7}$.

Tabella 1. Corpus

\begin{tabular}{lll}
\hline & Spagnolo L1 & Spagnolo L2 \\
\hline Dialoghi & $2\left(10^{\prime}+10^{\prime}\right)$ & $2\left(12^{\prime}+14^{\prime}\right)$ \\
Transaction & 84 & 51 \\
\hline
\end{tabular}

\subsection{Classificazione funzionale: mosse di introduzione e di gestione di un DT}

Per osservare e valutare le diverse modalità di introduzione e di gestione di un DT, ci serviamo di uno schema di annotazione pragmatica, Pr.A.T.I.D (Savy 2010), elaborato appositamente per l'annotazione di questo tipo di dialoghi.

Pr.A.T.I.D è uno schema multilivello che si basa su una caratterizzazione in mosse dialogiche (move) codificate attraverso etichette (tag). Ciascuna di esse indica la funzione comunicativa prevalente svolta dall'atto dialogico ${ }^{8}$. Riprendendo lo schema Map-task dell'HCRC di Edimburgo (Anderson et al. 1991, Isard \& Carletta 1995, Carletta et al. 1996, 1997), lo schema Pr.A.T.I.D si basa su una tripartizione della struttura dialogica in transaction, macrostrutture durante le

\footnotetext{
${ }^{7}$ Le differenze numeriche in termini di Transaction/dialogo verranno commentate tra i risultati; si veda $\S 4.2 .2$ ).

${ }^{8}$ Data la polifunzionalità intrinseca degli atti linguistici, molto spesso, chiaramente, una mossa non veicola una sola funzione comunicativa, ma più funzioni su piani diversi. Tuttavia, come nel caso di altri schemi ad una dimensione, lo schema impiegato si limita ad annotare la funzione che si considera principale rispetto allo sviluppo dell'interazione e allo specifico task.
} 
quali i partecipanti effettuano un primo passo per portare a compimento il task; conversational game, pezzi di dialogo suddivisi in base allo scopo che si vuol raggiungere e formanti le transaction; conversational move, enunciazioni minime prodotte dai parlanti e costituenti i game.

Nei dialoghi task-oriented è dunque possibile identificare, da un lato, una precisa struttura macrotestuale, -determinata appunto dalle varie transaction- ${ }^{-}$, dall'altro, una struttura testuale, caratterizzata dall'esplorazione dell'argomento all'interno di ciascuna transaction -articolata in uno o più game, a loro volta gestiti da uno scambio di mosse dialogiche.

L'esempio (1) mostra l'articolazione di due transaction che vertono sui DT estatua e coche (le cui aperture e chiusure sono indicate rispettivamente dalle etichette TR_Begin e TR_Closure). All'interno poi della prima transaction, possiamo osservare due aperture di game (etichetta Open), a loro volta gestiti da diverse mosse conversazionali.

(1)

p2 en tu estatua $</ T R \_$Begin $>$

p2 i el hombre tiene algo en la mano ?</Open>

p1 una espada $</$ End $>$

p2 en el mío también</End $>$

p1 ¿ y está montado en un caballo ?</Open>

p1 supongo que sí</End $>$

p2 sí</TR_Closure $>$

p1 el coche va a ten+ tiene se le ven las ruedas $i$ no ? $</ T R \_B e g i n>$

p2 sí</End $>$

p2 las dos de delante $</$ End $>$

p1 sí</TR_Closure $>$

Dati gli obiettivi del lavoro, consideriamo di seguito le mosse dialogiche, codificate dalle move-tag, che servono per introdurre e gestire un DT. La Tabella 2 illustra, per ciascun tipo di mossa, la sua funzione e una delle sue possibili realizzazioni ${ }^{9}$.

Tutte queste mosse possono sia introdurre che gestire un DT. Tuttavia, alcune si prestano meglio all'introduzione, altre alla gestione.

\footnotetext{
${ }^{9}$ Le mosse dialogiche sono codificate nello schema Pr.A.T.I.D dalle etichette sopra illustrate in base alla loro funzione comunicativa primaria. La codifica non dipende, quindi, in maniera diretta dalla forma che assumono, che può, infatti, essere variabile (sebbene esistano per ciascuna mossa forme più ricorrenti di altre).
} 
Tabella 2. Mosse dialogiche per introdurre e gestire un DT

\begin{tabular}{|c|c|c|}
\hline etichetta & funzione & esempio \\
\hline Action_directive & Direzione, conduzione & Fíjate en el hombre \\
\hline Open_option & Esortazione, proposta & $\begin{array}{l}\text { ¿Y si empezamos } \\
\text { por el hombre? }\end{array}$ \\
\hline Explain & $\begin{array}{l}\text { Fornitura di informazione, } \\
\text { descrizione }\end{array}$ & $\begin{array}{l}\text { El hombre está } \\
\text { sentado en un } \\
\text { banco }\end{array}$ \\
\hline Info_request & $\begin{array}{l}\text { Richiesta di contributo } \\
\text { generico }\end{array}$ & ¿Y el hombre? \\
\hline Query_w & $\begin{array}{l}\text { Richiesta di contributo } \\
\text { ampio }\end{array}$ & $\begin{array}{lll}\text { ¿Cómo } & \text { es } & \text { el } \\
\text { hombre } & \text { en } & \text { tu } \\
\text { dibujo? } & & \end{array}$ \\
\hline Query_y & $\begin{array}{l}\text { Richiesta di contributo } \\
\text { specifico }\end{array}$ & $\begin{array}{l}\text { ¿El hombre tiene el } \\
\text { pelo negro? }\end{array}$ \\
\hline Check & Richiesta di conferma & $\begin{array}{l}\text { Está como en una } \\
\text { plaza, ¿no? }\end{array}$ \\
\hline Align & Allineamento & Pues el hombre \\
\hline
\end{tabular}

La mossa info_request, ad esempio, è per lo più adatta all'introduzione di un DT, perché generalmente verte su un'entità nuova nel discorso. Tuttavia, potenzialmente potrebbe anche essere impiegata nella fase di negoziazione e gestione di un DT già dato. In questo caso, potrebbe servire per sollecitare ulteriore contributo, come in ¿y qué más? nell'esempio (2):

(2)

- El hombre está sentado en un banco

- Sí, sí, ¿y qué más? [info_request]

- se le ve solamente la pierna derecha

Altre mosse, invece, pur potendo anche introdurre un DT, generalmente vengono impiegate per gestirlo. Si pensi, ad esempio, alla mossa check, che codifica una richiesta di conferma. Essendo impiegata quando il parlante ha già un insieme abbastanza saldo di presupposizioni circa un DT, è più probabile che riguardi una entità topicale non nuova, bensì data nel discorso o anche "ratificata" (Lambrecht \& Michaelis 1998). 


\subsection{Analisi e interpretazione dei dati}

L'analisi esposta nel presente contributo consiste nell'investigazione delle strategie pragmatiche adottate dai parlanti per la risoluzione del task assegnato.

A tale scopo si è osservato il modo in cui viene proposta di volta in volta l'esplorazione di un nuovo argomento, quindi la sua introduzione, e l'organizzazione della struttura testuale che ne consegue durante la sua gestione attraverso lo sviluppo di eventuali catene topicali.

L'analisi si muove su un piano quantitativo e su un piano qualitativo e i dati raccolti vengono interpretati alla luce dei valori pragmatico-funzionali che le mosse dialogiche possono assumere su livelli diversi dell'interazione. In Savy \& Alfano (in press) viene infatti proposta un'interferenza di diversi fattori in gioco:

- il commitment, vale a dire la responsabilità che il parlante si assume nella conduzione del gioco;

- il coinvolgimento dell'interlocutore per lo svolgimento del compito;

- l'interazione, ovvero lo spazio che il parlante lascia al suo interlocutore ponendosi su un piano di scambio comunicativo;

- la "performatività", intesa come lo sforzo ed efficienza del parlante nel risolvere il compito scegliendo mosse immediatamente dirette alla sua soluzione;

- la cortesia linguistica, quindi l'attitudine del parlante a contenere la conflittualità e a favorire un'interazione armonica pur sacrificando la risoluzione del compito.

Tra tali fattori, alcuni (commitment, 'performatività') sono primari in questo tipo di scambio perché finalizzati direttamente al raggiungimento del task; gli altri (interazione, coinvolgimento e cortesia) sono comuni ad ogni scambio comunicativo e riguardano prevalentemente la gestione del rapporto tra gli interlocutori. Ciascuna mossa si caratterizza per presenza/assenza (ma anche grado) di un determinato parametro col quale contribuisce allo sviluppo del dialogo: in Tabella 3 sono riassunti i valori funzionali delle mosse di introduzione ${ }^{10}$.

\footnotetext{
${ }^{10} \mathrm{E}$ ' utile sottolineare che tali valori non provengono direttamente dall'analisi di un corpus, ma dalla riflessione sulle valenze delle mosse dello schema Pr.A.T.I.D: pertengono dunque a un livello astratto, categoriale la cui validità, tuttavia, è stata utilmente verificata in un numero consistente di lavori di analisi sviluppati dagli autori negli ultimi 10 anni. E' bene precisare anche che non si tratta di una matrice binaria, ma di una rappresentazione schematica di valori distribuiti su continua pragmatico-funzionali, in cui ciascuna mossa occupa un punto variabile
} 
Tabella 3. Caratterizzazione funzionale-pragmatica delle mosse (Savy \& Alfano in press)

\begin{tabular}{|l|c|c|c|c|c|}
\hline & Commitment & Coinvolgimento & Interazione & Performatività & Cortesia \\
\hline Action_directive & + & - & - & + & - \\
\hline Open_option & - & + & + & - & + \\
\hline Explain & + & - & - & \pm & \pm \\
\hline Info_request & - & + & + & \pm & + \\
\hline Query_w & - & + & - & + & + \\
\hline Query_y & - & - & - & + & - \\
\hline Check & + & - & - & + & \pm \\
\hline Align & - & \pm & + & \pm & + \\
\hline
\end{tabular}

Va da sé, come suggerito in Savy \& Alfano (in press), che le mosse più efficienti e dirette allo svolgimento del compito, siano anche quelle maggiormente 'autoreferenziali' per così dire, cioè quelle dotate di minor relazione con l'interlocutore. Le scelte del parlante, quindi, sono determinate dal gioco di questi fattori con alcune limitazioni che discuteremo tra breve.

Questo quadro interpretativo, inteso come 'strumento' di analisi, viene applicato ai dati che è stato possibile raccogliere con un'analisi quantitativa sviluppata secondo un percorso a tappe. In primo luogo è stato osservato il numero e il tipo di mosse utilizzato dai parlanti per l'introduzione di un nuovo topic al fine di individuare quali sono le mosse con maggiore e minore frequenza d'uso.

Inoltre, considerato che all'introduzione di un topic può seguire o meno un suo ulteriore approfondimento, è stata operata una distinzione fra transaction "chiuse" (TR-) e transaction "ramificate" (TR+). Nelle prime l'argomento non viene ulteriormente sviluppato, ma si esaurisce con mosse di chiusura da parte dell'interlocutore. Nelle seconde l'argomento introdotto viene invece esplorato dai parlanti, che ne gestiscono le informazioni attraverso successivi game dando origine ad una struttura testuale più complessa e gerarchicamente oraganizzata.

Successivamente, si è passato all'osservazione della gestione degli argomenti tramite lo studio delle mosse d'apertura di game, di cui sono stati analizzati il numero, i tipi e le funzioni.

tra due estremi (si veda Savy \& Alfano in press). Infine, esistono ovviamente differenze sull'uso e il valore accordato ad una mossa in lingue diverse (ad esempio, i parlanti di tedesco sembrano utilizzare il check con un valore aggiunto di cortesia, a differenza dell'italiano, si vedano House 2006; Schettino 2018). Tuttavia, le comparazioni fino ad ora effettuate tra italiano e spagnolo mostrano una buona coincidenza sul valore d'uso di questa mossa. 
Tale metodo d'analisi è stato applicato innazitutto su dialoghi prodotti da parlanti nativi di lingua spagnola (spagnolo L1) e in seguito su dialoghi prodotti da apprendenti di spagnolo come lingua straniera (spagnolo L2) di madrelingua italiana.

\section{Risultati: scelte strategiche e catene topicali}

\subsection{Spagnolo L1}

\subsubsection{L'introduzione del Topic}

L'analisi delle mosse d'apertura di transaction elicitate in spagnolo L1 permette di osservare quali vengano usate con maggiore frequenza e quali invece vengano dispreferite dai parlanti. Calcolando la percentuale di occorrenza di ciascuna mossa di apertura sul totale delle TR, otteniamo un quadro che evidenzia immediatamente il risultato di queste scelte, riportato in Figura 2.

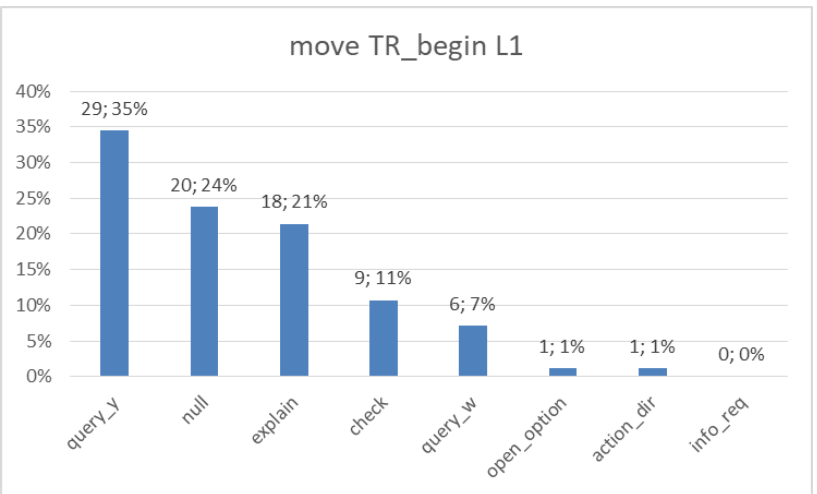

Figura 2. Mosse di apertura di transaction (numero di occorrenze; \%) in spagnolo L1

Come si evince dal grafico in Figura 2, le mosse di tipo query_y sono le preferite dai parlanti con il 35\% delle occorrenze, seguite dalle mosse di tipo align (24\%). L'elevato numero di mosse align utilizzate per l'introduzione di un nuovo topic è un dato atteso, difatti la loro funzione è proprio quella di attirare l'attenzione dell'interlocutore su un nuovo argomento introdotto. Tra le mosse dispreferite figurano invece open_option (1\%); action_directive (1\%) e info_request $(0 \%)$.

La strategia preferita dai parlanti, deducibile dall'analisi dei dati quantitativi, oscilla quindi tra un Questioning serrato (rappresentato da domande sì/no incentrate su ipotesi del parlante, Alfano \& Savy 2014) e un tentativo di 'allineare' 
l'interlocutore sull'argomento (o sul suo referente) attraverso richiami espliciti (mosse align) o impliciti al contenuto (che viene descritto da mosse di tipo explain).

Fatta eccezione per le mosse align, tale atteggiamento del parlante può essere letto, seguendo lo schema descritto in Tabella 3, come attenzione massima all'obiettivo di risoluzione del task e anche come assunzione di responsabilità e 'guida' del gioco da parte di chi introduce o 'apre' la partita: le mosse di tipo query_y infatti sono caratterizzate da un alto grado di 'performatività', mentre le mosse explain hanno un alto grado di 'commitment'. Questa scelta 'sacrifica' di conseguenza, nella modalità di proposta di un DT, la relazione aperta e positiva con l'interlocutore a vantaggio dell'efficienza nel compito, ma è mitigata dall'uso di align che, al contrario, puntano su una cortese preoccupazione di mantenere il contatto con l'altro. Come già osservato in Savy \& Alfano (in press) sono dispreferite, invece, le mosse che cumulano commitment e 'performatività' (action_dir) perché eccessivamente aggressive e mortificanti dell'interlocutore, così come quelle troppo deboli sul piano della 'performatività' (open_op, infor_req) in quanto abdicanti e poco efficienti, sebbene molto rispettose della relazione interpersonale ${ }^{11}$.

Non è scontato tuttavia che la strategia con cui il segmento dialogico viene aperto sia poi mantenuta nel corso dell'interazione e della gestione del DT, come vedremo in ciò che segue.

\subsubsection{La gestione del topic: articolazione e modalità}

Una volta introdotto un DT attraverso una scelta precisa del parlante che conduce una determinata tranche dell'interazione, la gestione di quell'argomento è più complicata da prevedere e da descrivere ricorrendo unicamente ad osservazioni di tipo quantitativo.

Innanzitutto occorre accertarsi che il DT introdotto o proposto venga 'accettato' dall'interlocutore, vale a dire che il suo status di argomento intorno al quale centrare una porzione di interazione sia sancito attraverso mosse specifiche che aprono successivi game all'interno della transaction. Come abbiamo accennato in $\S 3.3$, l'interlocutore può 'chiudere' immediatamente il game e provocare (o produrre egli stesso) un cambio di topic, oppure dimostrarsi disponibile ad esplorarlo attraverso nuovi game.

Come possiamo notare nella Figura 3, il numero di TR ramificate $(\mathrm{TR}+)$ è in maggioranza rispetto alle TR chiuse (TR-), che rappresentano comunque un terzo

\footnotetext{
${ }^{11}$ Savy \& Alfano (in press) sottolineano questo dato come strategia comune a diverse lingue esaminate, indipendentemente dalle scelte diverse effettuate localmente.
} 
del totale delle TR: vale a dire che in un terzo dei casi un topic proposto non è successivamente esplorato (come nell'esempio seguente).

(3)

$\begin{array}{ll}\text { p1 explain } & >\text { y al otro lado hay un edificio con dos chimeneas } \\ & </ \mathbf{T R} \_ \text {Begin }>\end{array}$

TR ramificate vs. chiuse L1

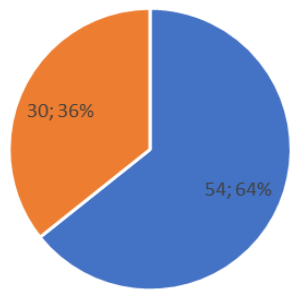

- $T R+$ ! TR-

Figura 3. TR ramificate $v s$. chiuse (numero di occorrenze; \%) in spagnolo L1

Negli altri due terzi dei casi, invece, si dà luogo ad una struttura testuale del dialogo articolata e sviluppata su più livelli. In Figura 4 possiamo osservare che il rapporto tra game e transaction è mediamente di 2:1 e sale a 3:1 se si considerano soltanto le transaction che ramificano. Tale media indica che l'esplorazione di un DT può impiegare un numero di game variabile da un minimo di 2 a un massimo di 14 game più o meno lunghi e più o meno articolati: il numero medio di mosse per transaction è infatti di 10,1 (con una media di 5 mosse per game e di 8,4 mosse al minuto).

Entrambi i parlanti possono collaborare (e di fatto collaborano) nell'esplorazione del DT proponendo e aprendo segmenti successivi di dialogo che approfondiscono e articolano un argomento attraverso numerosi game necessari a gestire lo scambio di informazioni su vari meronimi dell'oggetto osservato. 


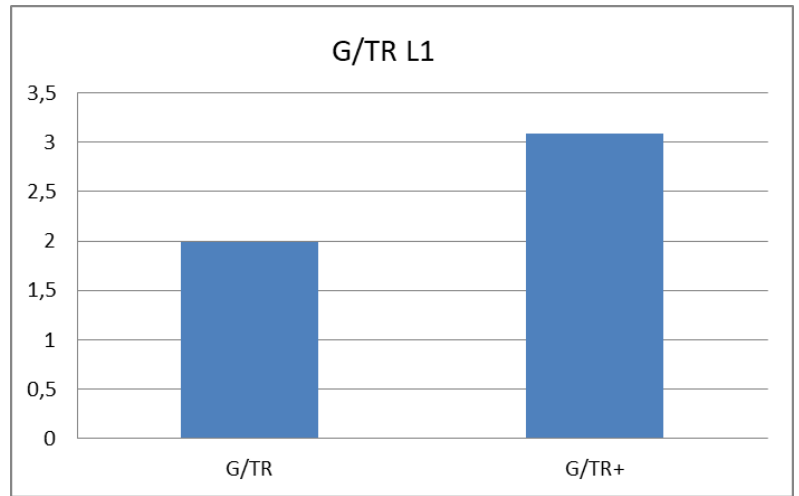

Figura 4. Rapporto tra game e transaction in spagnolo L1

A seguire è possibile osservare un esempio (4) di transaction sottoarticolata in game. Nella colonna a sinistra è indicato il parlante che ha prodotto l'enunciato, nella colonna centrale è riportata l'etichetta della mossa secondo lo schema Pr.A.T.I.D e nella colonna a destra è presente la trascrizione delle mosse del dialogo etichettate come mosse d'apertura o chiusura. In grassetto sono evidenziate le aperture di transaction (TR_Begin) e le aperture di game (Open).

(4)

p1 action_dir >pues seguimos con la casa</TR_Begin>

p2 acknowledge >vale</End $>$

p1 explain >la mía tiene ocho ventanas, que antes me he equivocado</Open>

p2 align >pensaba que ibas a decir las rayas del tejado</Open>

p1 reply > ah bueno es verdad a ver si las puedo contar $</$ End $>$

p2 explain >la mía creo que tiene siete</Open>

p1 acknowledge $>\mathrm{o}+$ sí</End $>$

p1 clarify $>$ siete u ocho $</$ End $>$

p2 explain >yo creo que siete</Open>

p1 object >yo creo que ocho, pero bueno</End $>$

p2 fatic > bueno ehm $<$ /End $>$

p1 acknowledge >sí, siete u ocho</End $>$

p1 over >es que no se ven muy bien $</$ End $>$ 


$\begin{array}{lll}\text { p1 } & \text { query_y } & >\text { idos chimeneas, una antena con dos rayas?</Open> } \\ \text { p2 } & \text { reply_y } & >\text { sí</End }> \\ \text { p2 } & \text { check } & >\text { y las chimeneas son bueno con un triangulito encima, } \\ & & \text { ino? }</ \text { Open> } \\ \text { p1 } & \text { reply_y } & >\text { sí</End }> \\ \text { p1 } & \text { clarify } & >\text { como una flecha }</ \text { End }> \\ \text { p2 } & \text { acknowledge } & >\text { sí</End }> \\ \text { p1 } & \text { explain } & >\text { luego, cinco ventanas en vertical }</ O p e n> \\ \text { p2 } & \text { object } & >\text { ehm no, la mía no tiene }</ \text { End }> \\ \text { p2 } & \text { hold } & >\text { te refieres a en a la parte de la derecha</End }> \\ \text { p1 } & \text { reply } & >\text { allí en horizontal, sí</End }> \\ \text { p1 } & \text { explain } & >\mathbf{y} \text { luego en vertical tres } \mathbf{y} \text { dos }</ \text { Open }> \\ \text { p2 } & \text { acknowledge } & >\text { vale, sí</End }> \\ \text { p2 } & \text { clarify } & >\text { la mía también }</ \text { End }>\end{array}$

I parlanti esplorano in maniera approfondita e articolata il topic introdotto attraverso numerosi games necessari a gestire lo scambio di informazioni su vari subtopic meronimi dell'oggetto osservato (casa $>$ finestre/tetto/comignoli).

Se però osserviamo ancor più in dettaglio le interazioni, notiamo come i game non siano tutti uguali, ma si differenzino a seconda della funzione svolta, originando una struttura testuale del dialogo stratificata, come nell'esempio che segue:

(5)

p1\#217: pues vas a ser que sí que hay ¿ <eh> ? <lp> pues ¿ cómo puede ser ? pues vamos a empezar otra vez \#<p2\#218><laugh $>\#$ por la estatua, empecemos por la estatua

p2\#218: \#<p1\#217><laugh $>\#<$ lp $>$ vale $<$ sp $>\{\langle$ NOISE $>$ a ver $\}$

p1\#219: a ver $\langle$ sp> yo veo un caballo

p2\#220: sí

p1\#221: <tongue-click> con un $\underline{\text { hombre }}$ <ee> encima <breath>

p2\#222: la sillita

p1\#223: la sillita $<\mathrm{sp}>$ tiene sombrero el hombre

p2\#224: 〈mhmh>

p1\#225: ¿ sí ?

p2\#226: sí

p1\#227: y espada <sp> \{<whispering > hemos dicho $i$ no ?\}

p2\#228: vale

p1\#229: larga 
p2\#230: y la mano izquierda coge al caballo $i$ no ?

p1\#231: sí y la mano derecha alzada con la espada

p2\#232: vale

p1\#233: la nariz puntiagu + , no tiene \#<p2\#234> ojos $<$ sp > ni boca\#

p2\#234: \#<p1\#233> no, el mío tampoco\#

La sezione di dialogo riportato corrisponde ad una transaction relativa all'immagine di una statua. Il DT estatua, introdotto in apertura della transaction, è gestito tramite il susseguirsi di vari game. Tra questi, alcuni introducono dei sub-topic (caballo, hombre, sillita), altri gestiscono ed esplorano i sub-topic introdotti (come nel caso di hombre) attraverso l'introduzione di meronimi del topic (sombrero, espada, mano izquierda, mano derecha, nariz, ojos, boca).

È possibile dunque individuare le diverse possibili funzioni dei game:

- gestione del DT proposto nella mossa di apertura di una transaction;

- introduzione di sub-topic;

- gestione di sub-topic con eventuale introduzione ad esplorazione di sub-subtopic, metonimici o meronimi del sub-topic;

- semplice scambio interazionale fra i parlanti non legato all'esplorazione del DT.

Vediamo ora quali siano le strategie di questa gestione: in Figura 5 notiamo che la distribuzione d'occorrenza delle mosse preferite dai parlanti varia rispetto a quelle scelte per aprire una transaction.

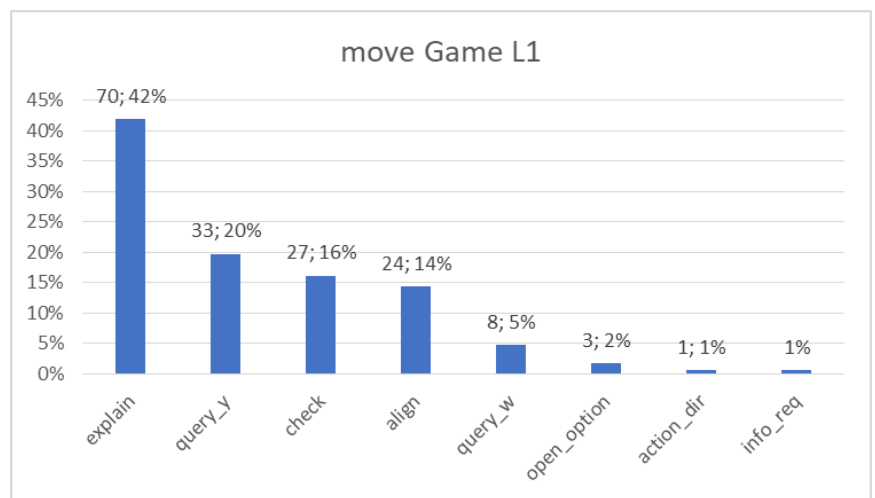

Figura 5. Mosse di gestione di transaction (numero di occorrenze; \%) in spagnolo L1 
La mossa con il maggior numero di occorrenze è l' explain (42\%), in cui il parlante non si preoccupa di elicitare un contributo da parte dell'interlocutore. A distanza troviamo le mosse query_y (20\%) e check (16\%), che richiedono un contributo informativo dell' interlocutore minimo, mentre retrocede al solo 14\% 1'occorrenza delle align.

In sostanza i parlanti non abbandonano le strategie finalizzate alla soluzione del task, anzi le accentuano a danno dell'interazione e dell'atteggiamento rispettoso dell'interlocutore, presi evidentemente dall'ansia della 'performance'. L'inversione di occorrenze tra explain e query_y è una possibile conseguenza dello sviluppo testuale in cui all'aumento dei dettagli della descrizione corrisponde un aumento delle mosse 'descrittive'.

Resta pure confermata la dispreferenza per strategie dialogiche eccessivamente forti o eccessivamente deboli (scarsità di action_dir, open_op e info_req), che evidentemente vengono considerate inefficaci.

\subsection{Spagnolo L2}

Passeremo ora in rassegna, utilizzando il medesimo schema metodologico e interpretativo, le produzioni di parlanti italofoni in spagnolo L2, mettendo direttamente a confronto i dati con quelli dei nativi.

\subsubsection{L'introduzione del Topic}

La Figura 6 mostra i risultati relativi all'analisi delle mosse scelte dagli apprendenti in L2 per proporre un nuovo argomento, a confronto con i risultati ottenuti per i parlanti nativi presentati nel precedente paragrafo.

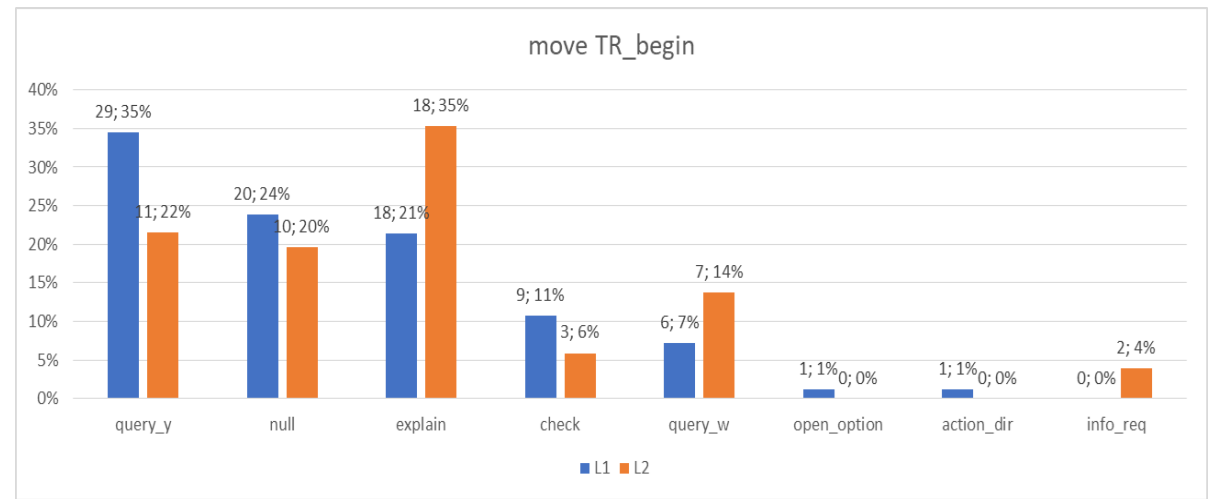

Figura 6. Mosse di apertura di transaction (numero di occorrenze; \%) in spagnolo L1 e L2 a confronto 
Nonostante la 'testa della classifica' non vari nella sua composizione, si può notare che, diversamente da quanto osservato in L1, il tipo di mossa preferita dagli apprendenti di spagnolo è l'explain, che presenta all'interno dei dialoghi in L2 una frequenza d'occorrenza del 35\% nettamente maggiore rispetto alle altre mosse impiegate, seguita dalla query_y utilizzata nel $22 \%$ dei casi. Un'altra differenza interessante è il maggiore impiego negli apprendenti di query_w, la cui frequenza è del $14 \%$ all'interno dei dialoghi in spagnolo L2 e del $7 \%$ in quelli in spagnolo L1, e di info_request che, pur poco presenti, sono invece completamente assenti nei parlanti nativi. Quest'ultime sono mosse volte all'elicitazione di un contributo più ampio e generico e dunque, in linea di principio, più impegnative per l'interlocutore.

Dovremmo quindi dedurre che l'apprendente scelga di introdurre un DT attraverso una mossa che implica un alto grado di responsabilità nell'interazione al pari di quanto fanno i madrelingua. Tuttavia, l'elevata occorrenza di explain e query_y può trovare una spiegazione diversa, considerando il piano delle competenze grammaticali, oltre che pragmatiche dei parlanti. Infatti, gli apprendenti, non possedendo ancora un pieno controllo linguistico sul dominio modale e lessicale della lingua target, potrebbero prediligere una strategia descrittiva basata sull'uso di strutture meno marcate come quella dichiarativa e interrogativa polare $^{12}$. Si metterebbe in atto così uno dei meccanismi tipici della 'competenza strategica', che interviene per far fronte alle difficoltà di comunicazione (Canale \& Swain 1980; Izquierdo 2005) $)^{13}$.

Inoltre, la scelta degli apprendenti di cedere maggior spazio di interazione al proprio interlocutore, attraverso l'uso relativamente maggiore di query_w e info_request (mosse poco performative, ma coinvolgenti dell'interlocutore) potrebbe risultare dalla messa in atto di una strategia pragmatica di 'deresponsabilizzazione' più che di cortesia (una sorta di 'passaggio di palla'), dovuta ad insicurezze relative alla loro competenza linguistica nella lingua target.

Si conferma anche in L2 la dispreferenza per strategie aggressive, mentre una mossa che abbiamo definito 'debole' come l'info_request, fa la sua comparsa a sostegno dell'ipotesi di insicurezza.

\footnotetext{
${ }^{12}$ Coerentemente con la Teoria della Processabilità (Pienemann 1998; Pienemann et al. 2005): le strutture dichiarative presentano, nella maggior parte dei casi, un ordine dei costituenti 'canonico'; in lingue come l'italiano e lo spagnolo, le interrogative polari sono realizzate esclusivamente attraverso il profilo tonale, senza modificare l'ordine dei costituenti. Tali strutture guidano la prima fase dello sviluppo sintattico dell'apprendente di L2 (si veda anche Bettoni \& Di Biase 2015).

${ }^{13}$ Previsto anche nel QCER (2002)
} 
Ulteriori dati a favore di questa ipotesi vengono dall'analisi dello sviluppo del dialogo che troviamo di seguito.

\subsubsection{La gestione del topic: frammentazione e riduzione opzionale}

L'articolazione del dialogo in L2 presenta caratteri molto diversi, come era ipotizzabile, rispetto a quella dei parlanti nativi.

In primo luogo, considerando la distinzione tra transaction chiuse e ramificate (Figura 7), vediamo che le prime ammontano a più della metà dei casi $(55 \%)$, mentre la frequenza d'occorrenza delle transaction ramificate scende notevolmente dal $64 \%$ di L1 al $45 \%$ in L2.

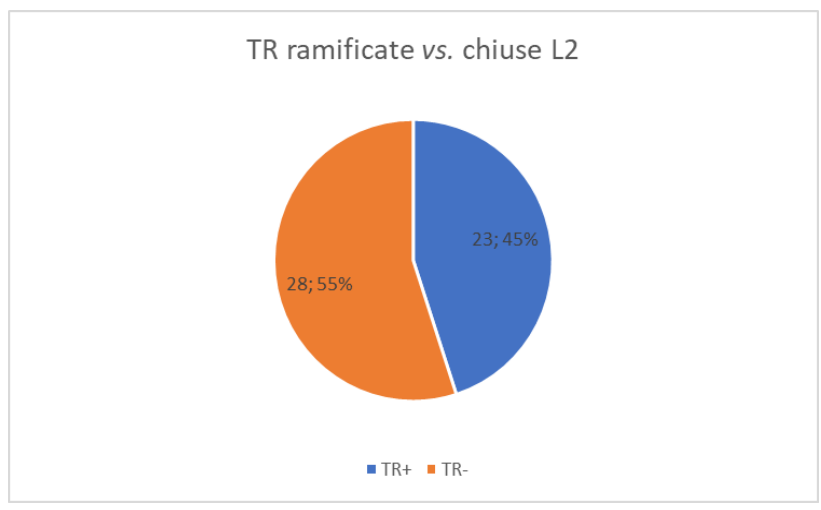

Figura 7. TR ramificate $v s$. chiuse (numero di occorrenze; \%) in spagnolo L2

Gli apprendenti tendono dunque a non approfondire l'esplorazione di un DT, generando una struttura testuale del dialogo molto più frammentata rispetto a quella rilevata in L1. Un esempio è riportato in (6):

(6)

$\begin{array}{lll}\text { p2 } & \text { explain } & \text { la televisión tiene ocho tastos }</ \text { TR_Begin }> \\ \text { p1 } & \begin{array}{l}\text { acknowledge } \\ \text { sí }</ \text { End }>\end{array} \\ \text { p1 } & \text { clarify } & \text { negros y tres blancos }</ \text { End }> \\ \text { p2 } & \begin{array}{l}\text { repeat reph- } \\ \text { rase }\end{array} & \text { tres blancos }</ \text { End }> \\ \text { p2 } & \begin{array}{l}\text { acknowledge } \\ \text { p1 }\end{array} & \text { sí }</ \text { End }> \\ & & \text { perocessed }</ \text { End }>\end{array}$




\begin{tabular}{|c|c|c|}
\hline $\mathrm{p} 2$ & fatic & y, no sé</End $>$ \\
\hline $\mathrm{p} 2$ & query_y & $\begin{array}{l}\text { ¿ las orejas del hombre son muy grandes } \\
?</ \text { TR_Begin }>\end{array}$ \\
\hline $\mathrm{p} 1$ & reply_y & sí, claro</End $>$ \\
\hline $\mathrm{p}^{\prime}$ & fatic & $\mathrm{y}</$ End $>$ \\
\hline $\mathrm{p} 1$ & null & no mira</TR_Begin> \\
\hline $\mathrm{p} 1$ & explain & $\begin{array}{l}\text { el caballo tiene un pie levantado y uno en el már- } \\
\text { mol</TR_Begin }>\end{array}$ \\
\hline ? & acknowledge & sí</End $>$ \\
\hline $\mathrm{p} 2$ & continue & apoyado a la estatua $</$ End $>$ \\
\hline & acknowledge & sí</End $>$ \\
\hline
\end{tabular}

Questo scambio evidenzia come gli apprendenti tendano a produrre transaction che si esauriscono subito con mosse di chiusura senza ramificare e svilupparsi in game. Il rapporto tra numero di game e di transaction è quindi quasi la metà che in lingua nativa (Figura 8), con una media di 1,25 game per transaction.

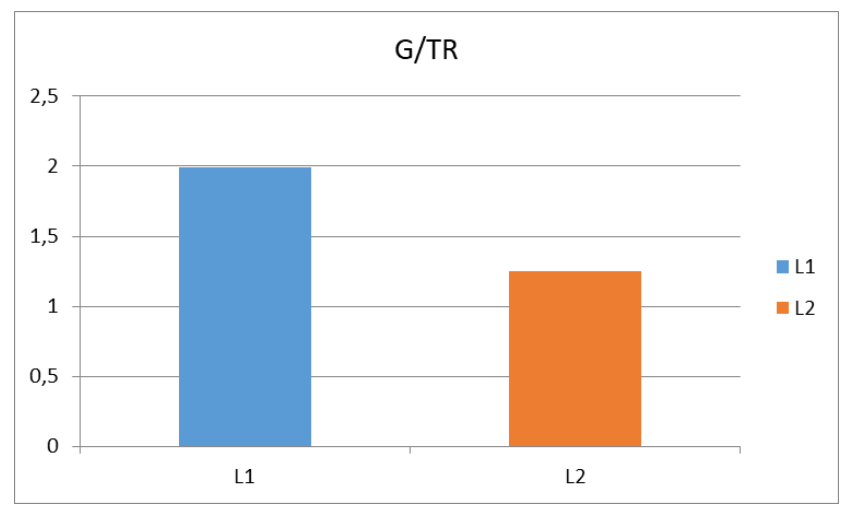

Figura 8. Rapporto tra game e transaction in spagnolo L1 e L2 a confronto

Ciò sta ad indicare che qualora il DT introdotto in una transaction venga esplorato, tale esplorazione non risulta particolarmente approfondita ed è comunque limitata a pochi game che si limitano alla gestione del topic proposto in apertura di transaction e al massimo all'introduzione di eventuali sub-topic. Tale risultato viene confermato dall'osservazione (Figura 9) del numero di mosse per transac- 
tion (9.3) che si approssima a quello di mosse per game (7.3). In sostanza si riscontra che nei dialoghi L2 le transaction ramificate non solo risultano in numero minore rispetto alla L1, ma sono anche meno articolate.

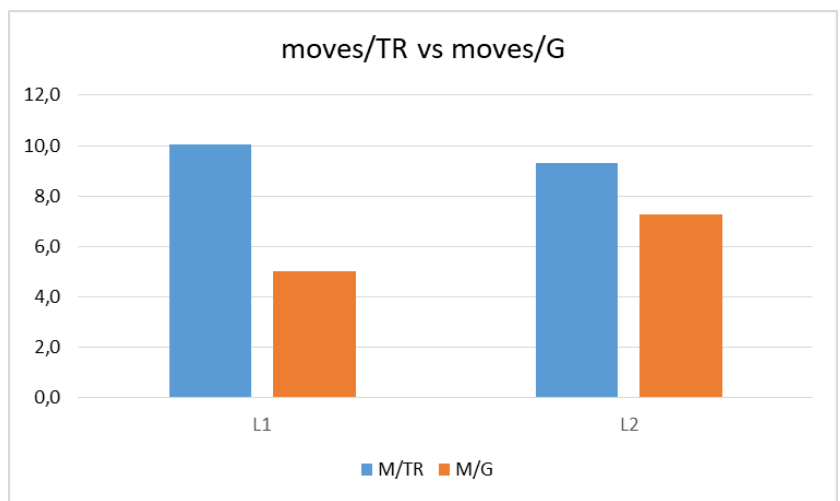

Figura 9. Numero di move per TR e numero di move per game in spagnolo L1 e L2 a confronto

Un secondo dato interessante nel confronto L1/L2 si evince da un'analisi più approfondita della 'durata' delle transaction (Figura 10): nonostante la maggioranza di esse in L2 non ramifichi e/o sia costituita da pochi game, la loro durata assoluta è sempre maggiore che in lingua nativa (di pochi secondi in media per le TR chiuse, di $20 \mathrm{sec}$ in media per quelle ramificate).

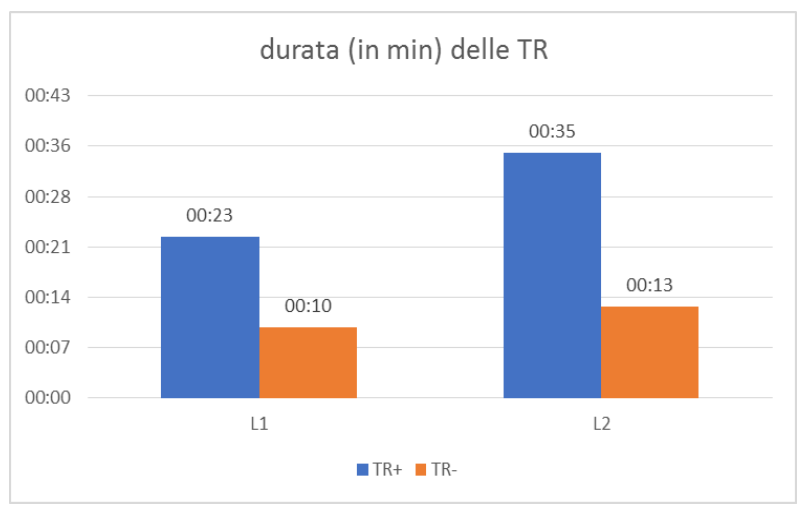

Figura 10. Durata delle TR in spagnolo L1 e L2 a confronto 
Ciò correla da un lato con una minore velocità d'elocuzione, ossia una maggiore lentezza dell'enunciazione (come si evince dalla Figura 11a), e dall'altro con una minore fluenza e un'alta incidenza di fenomeni di esitazione e di pause piene e vuote (il doppio in L2 rispetto alla L1, con un'incidenza di 3 volte maggiore rispetto al numero di parole, come risulta dai dati in Figura 11b). Entrambi possono essere ragionevolmente considerati come indici di lentezza di elaborazione e difficoltà di gestione dell'interazione.

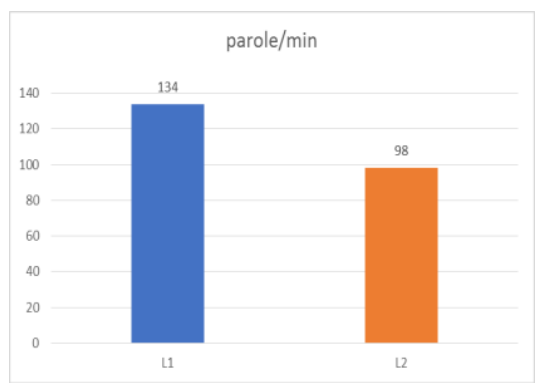

(a)

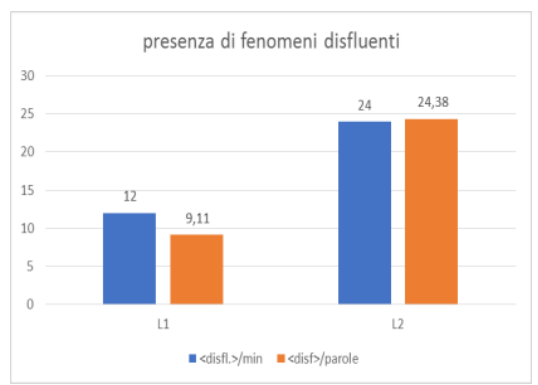

(b)

Figura 11. Velocità (a) e fluenza (b) in spagnolo L1 e L2 a confronto

L'osservazione delle strategie elette dagli apprendenti (Figura 12) è conferma dello stato di insicurezza e delle difficoltà comunicative in L2: la mossa explain, attraverso la quale si descrive il contenuto della vignetta e gli elementi che la compongono, schizza al 66\% delle occorrenze totali, a danno di tutte le altre mosse opzionabili, ivi compresa la query_y (che scende al 14\%).

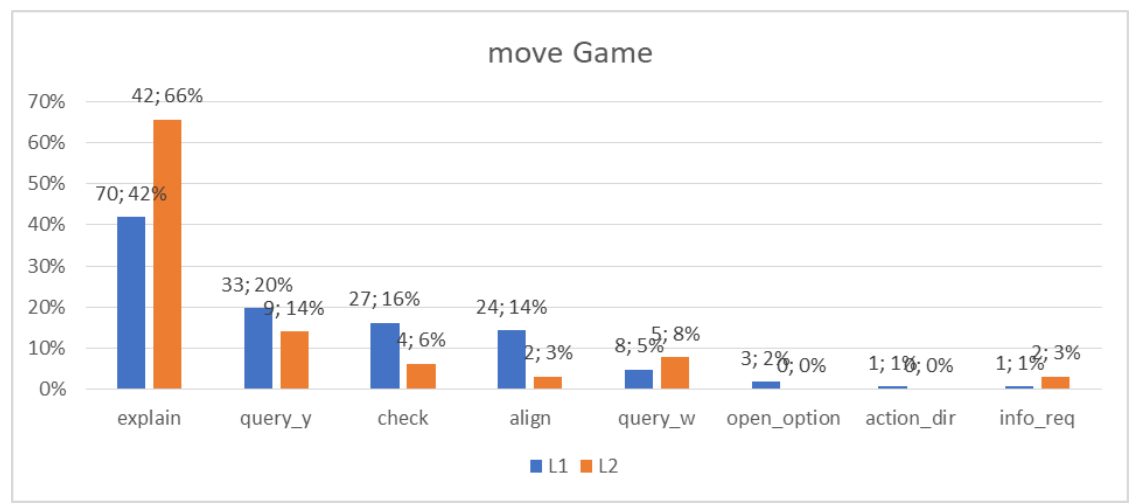

Figura 12. Mosse di gestione di transaction (numero di occorrenze; \%) in spagnolo L1 e L2 a confronto 
Non c'è dunque attenzione al piano dell'interazione, al coinvolgimento dell'altro, alla cortesia, ma nemmeno è presente lo sforzo performativo, in un compito in cui evidentemente il task oggettivo (la scoperta delle differenze del gioco) è superato da un task soggettivo, il quale presenta un livello di difficoltà superiore perché coinvolge il piano della stessa produzione verbale in L2. La scelta dell' explain non è una scelta strategica motivata dal gioco, ma una scelta "obbligata" dalla scarsa competenza del codice.

\section{L'Interazione e la negoziazione delle strategie}

Quanto abbiamo visto fino ad ora prosegue, come abbiamo detto, dall'osservazione ed elaborazione di dati frequenziali relativi a ciascuna mossa impiegata per introdurre e argomentare intorno ad un elemento topicale del discorso, considerato in maniera "statica" (o per meglio dire, indipendente dalla dinamica sequenziale che si instaura tra le mosse). Le interpretazioni che abbiamo avanzato, dunque, fanno riferimento a fattori pragmatici in gioco (il commitment, la cortesia, ecc.) che, tuttavia, vanno anch'essi contestualizzati tenendo presente il reale sviluppo dell'interazione: ogni fattore rappresenta un'opzione elegibile dal parlante, ma deve essere e viene 'negoziato' continuamente tra i due interlocutori in maniera implicita. Lo scambio dialogico, infatti, si configura come un'interazione sequenziale, in quanto si sviluppa nel tempo, e dinamica (Bazzanella 2002), poiché basata su un processo di costante negoziazione e adattamento reciproco tra i due interlocutori (Weigand \& Dascal 2001).

Vanno dunque analizzate le reazioni ad una determinata scelta strategica del parlante e l'effetto che tale reazione a sua volta ha sull'evoluzione del dialogo e sull'impiego delle strategie. Una particolare scelta strategica del parlante può infatti essere accettata o meno dal suo interlocutore, reazione che eventualmente influisce sulle scelte successive generando cambiamenti di strategia.

Osserviamo, ad esempio, il seguente scambio tra due parlanti madrelingua spagnoli:

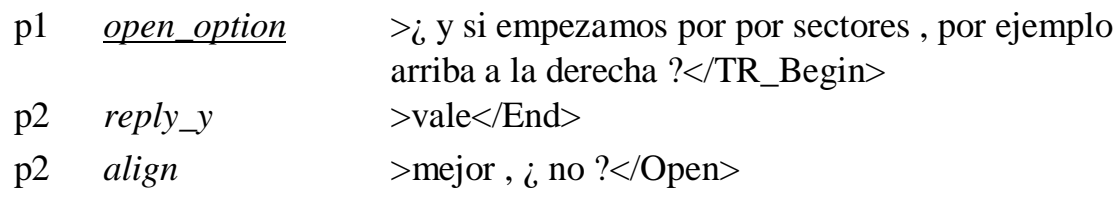


p1 align

$\mathrm{p} 2$ acknowledge

p1 acknowledge

p1 action_dir

p2 align

p1 acknowledge

p2 query_w

p1 acknowledge

p1 query_w

p1 reply_w

p2 acknowledg

p2 repeat_rephrase

p2 acknowledge

p2 clarify

p1 check

p2 check

p1 reply_y

p2 acknowledge
$>$ lo que , ¿ no ?</Open>

$>$ vale $</$ End $>$

$>$ vale $</$ End $>$

$>$ pues di por dónde empezamos $</$ Open $>$

$>$ a ver, pues, a la izquierda eeh bueno en la parte de arriba que está el árbol</Open $>$

$>$ sí sí</End $>$

$>$ eeh bueno cómo es el árbol de</Open $>$

$>$ vale $</$ End $>$

$>_{\text {¿ } \text { cuántas partes? }</ O p e n>}$

$>$ a ver yo veo a mano derecha del árbol como dos dos capas y a mano izquierda una por arriba y dos por abajo $</$ End $>$

$>$ sí</End $>$

$>$ dos partes $</$ End $>$

$>$ vale $</$ End $>$

$>$ la mía el mío también</End>

$>_{\text {¿ sí } ?</ O p e n>}$

$>$ y el tronco es finito, $i$ verdad $?</$ Open $>$

$>$ sí</End $>$

$>$ vale $</$ TR_Closure $>$

Un'analisi delle 'coppie adiacenti' (Schegloff \& Sacks 1973, Sinclair \& Coultard 1975) di enunciati in apertura di transaction o di game mostra indirettamentese la strategia scelta da un parlante dà i suoi frutti, viene accettata e condivisa e si mostra utile alla prosecuzione dello scambio o se invece ne viene richiesto l'abbandono o la riformulazione dalla reazione dell'interlocutore.

In (7), il p1 apre la transaction con una mossa open_option richiedendo così un contributo al p2, rafforzato dalla mossa align successiva (lo que) con cui verifica la disponibilità dell'interlocutore attendendosi ancora una volta che collabori attivamente. Dal momento che p2 non reagisce secondo le attese, p1 si ritrova costretto a cambiare strategia passando ad una action_directive, un imperativo che obbliga il secondo parlante a svolgere l'azione richiesta. In questo caso una scelta cortese e coinvolgente, sulla base di una reazione poco cooperativa, viene sostituita da una strategia di comando. 
Successivamente $\mathrm{p} 2$ elicita un contributo ampio da $\mathrm{p} 1$ con una query_w (bueno cómo es el árbol); tuttavia la risposta che ne riceve consiste in un'ulteriore domanda query_w (¿cuántas partes?) cui segue una spiegazione di questa strategia inattesa (a ver yo veo a mano derecha del árbol como dos dos capas y a mano izquierda una por arriba y dos por abajo). Il p1 spiega infatti il perché stia ponendo a sua volta una domanda, probabilmente consapevole del fatto che la mancanza di una risposta diretta alla domanda ricevuta possa generare un'incomprensione nel suo interlocutore. A seguito di questo episodio, p2 sceglie di cambiare strategia e utilizzare una mossa check per continuare l'esplorazione del topic. In questo modo riduce notevolmente lo spazio lasciato all'interlocutore chiedendogli di fornire una semplice conferma e assicurandosi una maggiore probabilità che la risposta ottenuta sia quella che voleva effettivamente elicitare.

$\mathrm{Si}$ osserva quindi un cambiamento strategico in entrambi i parlanti che è risultato dell' adattamento reciproco alle reazioni ottenute, in altre parole derivante dalla negoziazione della strategia messa in atto. La 'performatività' diventa così non solo materia della singola scelta, ma principio fondante dell'intera strategia di interazione: perseguire su una strada non condivisa vuol dire infilarsi in un vicolo cieco che non aiuterà il raggiungimento dell'obiettivo e necessita quindi una rivalutazione (ed eventuale abbandono) da parte del parlante.

\section{Discussione}

L'analisi condotta si riaggancia ai risultati di uno studio precedente (Savy \& Alfano in press) in cui veniva sperimentato un metodo che coniugasse l'osservazione di dati quantitativi estratti da un corpus con l'interpretazione delle strategie avanzata ricorrendo a fattori pragmatico funzionali di classificazione delle mosse utilizzate dai parlanti.

Riassumendo quanto riportato nei precedenti paragrafi, abbiamo osservato innanzitutto che i parlanti madrelingua spagnoli ${ }^{14}$ tendono a introdurre nuovi $\mathrm{Di}$ scourse Topic preferendo mosse con un alto grado di performatività volte all'immediata risoluzione del compito (query_y). Tale preferenza è indicativa del fatto che essi tendono ad assumere un ruolo guida nella risoluzione del task. Risultano invece "dispreferite" mosse volte all'elicitazione di un contributo generico, come le info_request, mosse che esprimono una semplice proposta e si caratterizzano per un'ancor maggiore debolezza performativa, come le open_option, e mosse che, di contro, presentano allo stesso tempo un forte

\footnotetext{
${ }^{14}$ Ovviamente, come già ribadito, siamo consapevoli che si tratta di un piccolo campione di parlanti, che non consente generalizzazioni estreme o assolute dei risultati.
} 
commitment e valore performativo e realizzano quindi una strategia troppo dominante e aggressiva, poco cortese e pertanto potenzialmente dannosa per la relazione fra interlocutori, come le action_directive.

Diversamente, gli apprendenti di spagnolo L2 hanno preferito l'impiego della mossa explain, che non presenta una particolare valenza performativa, ma è indice di un buon grado di commitment del parlante, il quale si assume un certo impegno nella risoluzione del compito, senza tuttavia richiedere esplicitamente la partecipazione dell'interlocutore. Abbiamo però attribuito a questa scelta un valore 'negativo', considerandola indice di scarso dominio modale e lessicale della lingua target. Insieme a questa mossa infatti, vengono introdotte query_w e info_req come strategie 'deresponsabilizzanti' piuttosto che coinvolgenti.

E' risultato anche evidente, dal confronto fra transaction "chiuse" (TR-) e transaction "ramificate" (TR+), che i dialoghi in L2 tenuti in L1 presentano una struttura meno elaborata e articolata e più frammentata rispetto a quelli in L1.

Per quanto riguarda la gestione dei DT nei game, sia nei dialoghi L1 che in L2 si è riscontrato un ampio impiego di explain: tuttavia nel primo caso risultano presenti anche un buon numero di mosse performative come le query_y e le check, per cui i parlanti ripropongono la già osservata tendenza ad assumere strategie di controllo; mentre in L2 l'explain è praticamente, se non l'unica, la mossa predominante a scapito di ogni altra opzione. L'assunto per cui l'elevata presenza di mosse poco coinvolgenti in L2, per tanto "egocentriche", sia dovuta a motivi di competenza linguistica, o meglio grammaticale, piuttosto che alla scelta di una vera e propria strategia di controllo, è confermato dal fatto che gli apprendenti prediligano una strategia descrittiva che permette un'efficacia funzionale commisurata alle risorse linguistiche a disposizione

Una considerazione qualitativa del modo in cui gli argomenti del discorso possono essere introdotti ed esplorati in spagnolo L1 ha permesso di osservare che i game svolgono differenti funzioni, come si è mostrato nell'esempio (4), riportato in $\S 4.1$. I parlanti sembrano quindi procedere utilizzando una strategia che potremmo definire "a cannocchiale", in cui, come in uno zoom, esplorano livelli di dettaglio di volta in volta più profondi, dando origine così ad una catena topicale strutturata gerarchicamente. Tale livello di dettaglio di esplorazione diminuisce notevolmente se si osservano i dialoghi prodotti in L2.

Infine, anche il dato relativo alla durata effettiva dell'interazione dialogica risulta riconducibile alla minore padronanza linguistica degli apprendenti. Essi necessitano infatti di più tempo per la scelta e messa in atto di una strategia pragmatica adeguata agli strumenti linguistici di cui dispongono, motivo per cui producono un numero maggiore di pause e di silenzi. 
In conclusione, la scelta strategica dei parlanti nativi e degli apprendenti di spagnolo per la risoluzione del compito assegnato differisce sostanzialmente, piuttosto che per motivi pragmatico-culturali, per questioni di competenza linguistica, in particolar modo grammaticale e lessicale.

Gli apprendenti dimostrano dunque di venir meno al rispetto delle "massime" comportamentali che gli interlocutori coinvolti in scambi dialogici di tipo taskoriented sembrano condividere (Savy \& Alfano in press) adottando per lo più una strategia descrittiva, connotata da un buon grado di impegno, ma non decisamente efficace, in quanto può essere realizzata attraverso strutture linguistiche elementari, e di deresponsabilizzazione più che di cortesia, risultante dalla tendenza a cedere lo spazio d'interazione e la responsabilità di conduzione del gioco, originando una struttura dialogica più frammentata e un'esplorazione degli argomenti limitata, meno dettagliata.

Infine, possibili sviluppi di quest' indagine potrebbero gettare luce sugli effetti della negoziazione delle strategie tra i parlanti. Questo tipo di analisi, effettuata per ora a campione su porzioni di dialoghi in L1, merita ulteriori indagini per realizzare uno studio longitudinale, che tenga conto quindi della natura sequenziale e dinamica del dialogo, che osservi le scelte operate dall'altro come un fattore di variabilità delle strategie messe in atto (Savy \& Alfano in press) e la maniera in cui esse evolvono nel tempo, lungo le differenti fasi dell'interazione.

\section{Riferimenti bibliografici}

Alfano, I. \& Savy, R. 2014. La strategia del questioning nell'interazione dialogica: verso una definizione di continua pragmatico-funzionali. Lingue e Linguaggi 12: 7-22.

Anderson, A.H., Bader, M., Bard, E.G., Boyle, E., Doherty, G., Garrod, S., Isard, S., Kowtko, J., McAllister, J., Miller, J., Sotillo, C., Thompson, H.S. \& Weinert, R. 1991. The HCRC Map Task Corpus. Language and Speech 34: 351-366.

Asher, N. 2004. Discourse topic. Theoretical Linguistics 20: 163-201.

Bazzanella, C. 2002. Sul dialogo. Contesti e forme di interazione verbale. Milano: Guerini e associati.

Bazzanella, C. 2015. Dimensione interculturale e prospettiva pragmatica della lingua. Alcune riflessioni tra teoria e applicazione. Segno 2,11-16.

Bettoni, C. \& Di Biase B. (eds) 2015. Grammatical Development in Second Languages: Exploring the Boundaries of Processability Theory. EUROSLA Monographs Series 3. Amsterdam: The European Second Language Association.

Beyssade, C. \& Marandin, J. 2002. Topic marking, discourse topic and discourse moves, in Bende Farkas A., Workshop on Information Structure in Context, Stuttgart, Germany.

Brown, G. \& Yule, G. 1983. Discourse Analysis. Cambridge: Cambridge University Press.

Büring, D. 1997. The meaning of topic and focus: the 59th street bridge accent, London: Routledge. 
Canale, M. \& Swain, M. 1980. Theoretical bases of communicative approaches to second language teaching and testing, Applied Linguistics 1(1), 1-47.

Carletta, J., Isard, A., Isard, S., Kowtko, J., Doherty-Sneddon, G. \& Anderson, A.H. 1996. HCRC Dialogue structure coding manual. Technical Report, 82. Human Communication Research Center, University of Edinburgh.

Carletta, J., Isard, A., Isard, S., Kowtko, J., Doherty-Sneddon, G. \& Anderson, A.H. 1997. The reliability of a dialogue structure coding scheme. Computational Linguistics 23(1): 13-32.

Cerrato, L. 2007. Sulle tecniche di elicitazione di parlato semispontaneo. Technical Report, progetto CLIPS. http://www.clips.unina.it (accessed February 20, 2018).

Chang, V. 2007. A Cognitive-pragmatic Approach to Discourse Topic: A Cross-linguistic Analysis and Universal Account. Journal of Universal Language 8(1): 1-21.

Chini, M. (ed.) 2010. Topic, struttura dell'informazione e acquisizione linguistica. Milano: FrancoAngeli.

Consiglio d'Europa (2002). Quadro comune europeo di riferimento per le lingue: apprendimento, insegnamento, valutazione. Firenze: La Nuova Italia-Oxford.

Cutugno, F., 2007. Criteri per la definizione delle mappe, esempi di mappe e di vignette per il gioco delle differenze. Clips-Corpora e Lessici di Italiano Scritto e Parlato, Technical Report, progetto CLIPS. http://www.clips.unina.it/it/documenti.jsp (accessed February 10, 2018)

De Leo, S. 2008. La struttura topicale in dialoghi task-oriented. In M. Voghera (ed), Testi e linguaggi 2, Roma: Carocci, 105-129.

Gundel, J. 1988. Universals of topic-comment structure. In M.Hammond, E. Moravcsik \& J. Wirth (eds), Studies in syntactic typology, Amsterdam: John Benjamins, 209-239.

Gundel, J. 2003. Information structure and referential givenness/newness. How much belongs in the grammar?. Journal of Cognitive Science 4: 177-199.

Gundel, J., Hedberg, N. \& Zacharski, R. 1993. Cognitive status and the form of referring expressions in discourse. Language 69: 274-307.

House, J. 2006. Communicative styles in English and German. European Journal of English Studies 10(3): 249-267.

Isard, A. \& Carletta, J. 1995. Transaction and action coding in the Map Task Corpus. Research Paper HCRC/RP-65.

Izquierdo Gil, M.C. 2005. La selección del léxico en la enseñanza del español como lengua extranjera. Colección Monografías 8, Málaga, ASELE.

Lambrecht, K. \& Michaelis, L. 1998. Sentence accent in information questions: default and projection. Linguistics \& Philosophy 21(5): 477-544.

Lambrecht, K. 1994. Information structure and sentence form: topic, focus and the mental representation of discourse referents. Cambridge: Cambridge University Press.

Malouf, R. 1995. Towards an analysis of multi-party discourse, http://citeseerx.ist.psu.edu/viewdoc/summary?doi=10.1.1.8.4024 (accessed Genuary 3, 2016).

Péan, V., Williams, S. \& Eskenazy, M. 1993. The design and recording of ICY, a corpus for the study of intraspeaker variability and the characterisation of speaking styles. Eurospeech '93, $3^{\text {rd }}$ European Conference on Speech Communication and Technology, Berlin, Germany, September 22-25, 627-630. 
Pienemann, M. 1998. Language Processing and Second Language Development: Processability Theory. Amsterdam: John Benjamins. ISBN 978-1-55619-549-5.

Pienemann, M., Di Biase, B. \& Kawaguchi, S. 2005. Extending Processability Theory. In M. Pienemann (ed), Cross-linguistic Aspects of Processability Theory. Amsterdam: Benjamins, 199-251.

Prévot, L. 2001. Topic structure in route explanation dialogues. Proceedings of the workshop: Information Structure, Discourse Structure and Discourse Semantics of the $13^{\text {th }}$ European Summer School in Logic, Language and Information, University of Helsinki, Finland, 145159.

Roberts, C. 1996. Information Structure in Discourse: Towards an Integrated Formal Theory of Pragmatics. In J. H Yoon. \& A. Kathol (eds), OSU Working Papers in Linguistics 49, Papers in Semantics, Ohio State University, Columbus, 91-136.

Savy R. \& Cutugno F. 2009. CLIPS. Diatopic, diamesic and diaphasic variations in spoken Italian. In Mahlberg M., González-Díaz V. \& Smith C. (eds.), Proceedings of the $5^{\text {th }}$ Corpus Linguistic Conference, CL2009, Liverpool, UK: 1-24.

Savy, R. \& Alfano, I. in press. Strategie pragmatiche di introduzione di un Topic Discorsivo in dialoghi task-oriented: lingue a confronto. Lingua in contesto: la prospettiva pragmatica.

Savy, R. \& Solís, I. 2008. Strategie pragmatiche in italiano e spagnolo a confronto: una prima analisi su corpus. In M. Voghera (ed), Testi e linguaggi 2, Roma: Carocci, 214-242.

Savy, R. 2010. Pr.A.T.I.D: a coding scheme for pragmatic annotation of dialogues. In N. Calzolari et al. (eds), Proceedings of the $7^{\text {th }}$ International Conference on Language Resources and Evaluation, LREC 2010, Valletta, Malta, May 17-23, 2141-2148.

Schettino, L., 2018. Le richieste d'informazione in tedesco: prospettiva pragmatica e prosodicalaspetti di interfaccia pragmatica-prosodia. Tesi Magistrale non pubblicata, Università di Salerno.

Sinclair, J.M. \& Coulthard, M. 1975. Towards an analysis of discourse: the English used by teachers and pupils. London: Oxford University Press.

Solís, I. \& Savy, R. 2012. Diferentes estrategias comunicativas en diálogos task-oriented españoles e italianos. In A. Cassol, F. Gherardi, A. Guarino, G. Mapelli, F. Matte Bon \& P. Taravacci (eds), Il dialogo. Lingue, letterature, linguaggi, culture. Roma: AISPI Edizioni, 443-457.

Weigand, E. \& Dascal, M. 2001. Negotiation and Power in dialogic interaction. Amsterdam/Philadelphia: Benjamins. 